\title{
AVISO
}

Por no disponer de los correspondientes originales informáticos, la maquetación de este artículo difiere de la del publicado en papel. Por lo demás, los contenidos no han sufrido ninguna alteración.

Artículo publicado en el fascículo $1^{\circ}$ del tomo LXV (1997) de EMERITA, pp. 57-63

Autor: Josep Antoni Clúa Serena

\section{NOTAS CRÍTICAS AL TEXTO DE LA ALEJANDRA DE LICOFRÓN}

Some suggestions are made on the text of the Alexandra by the author, who has just edited this work of Lycophron with introduction, notes and Catalan translation. He proposes some emendations and justifies his choices.

Con el fin de poder justificar algunas lecturas adoptadas frente a las ediciones de la Alejandra de Licofrón a cargo de L. Mascialino ${ }^{1}$ y de M. Fusillo- A. Hurst - G. Paduano ${ }^{2}$, publicada en fecha muy reciente, he tenido que plantearme algunas cuestiones textuales al abordar la tarea de edición y traducción (al catalán) de dicho poema helenístico ${ }^{3}$. Ofrezco, pues, un breve elenco de notas críticas al texto mencionado.

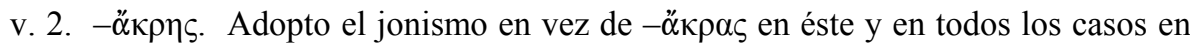
que la tradición manuscrita ofrece variantes dialectales diferentes al ático, dada la conocida apertura de la lengua de la tragedia griega con respecto a otros dialectos. Sigo en este punto el criterio de A. Hurst (cf. Licofrone. Alessandra p. 54), dado el «aspetto manierista del testo».

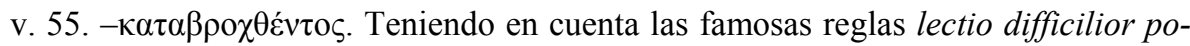
tior y recentiores deteriores (la segunda propugnada por Lachmann), y consciente de que no puede haber un mero error en la lectura (cf. la adoptada por los manuscritos deteriores, a saber - $\alpha \alpha \tau \alpha \beta \rho \omega \theta \dot{\varepsilon} v \tau o \varsigma$, que, sin embargo, L.Mascialino prefirió para su edición), me veo inclinado a adoptar esta lectura. Por otro lado, A.Hurst señala

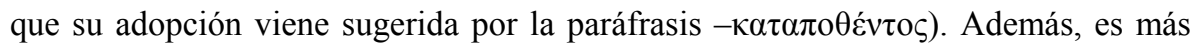
plausible la conjetura de Müller al verso 742 de la Alejandra, no aducida por Hurst, en la que se propone $-\kappa \alpha \tau \alpha \beta \rho o ́ \xi \eta$, es decir el mismo verbo que postulo para el verso

1 Cf. L. Mascialino, Lycophronis Alexandra, Leipzig, 1964; Idem, Licofrón. Alejandra, Barcelona, 1956.

2 Cf. Licofrone. Alessandra, Milán, 1991. Las notas críticas al texto pertenecen a A. Hurst. Esta edición no cuenta con aparato crítico, si bien explicita las lecturas adoptadas en su «Premessa al testo» (pp. 53-56).

3 Cf. Licòfron. Alexandra, Barcelona, 1995. 
que nos ocupa, en vez de - $\alpha \alpha \tau \alpha \beta \rho \dot{\xi} \eta$.

v. 64. $-\dot{\alpha} v \theta$ o $\pi \lambda i ́ \tau$ ov. Es mucho más plausible suponer un «adversario en las armas», referido a Filoctetes con respecto a Paris, que no un - $\alpha \dot{\theta} \theta 0 \pi \lambda i$ tov (Filoctetes tendría la «misma» arma que Paris, como propone A.Hurst, apoyándose en la lectura de varios manuscritos.

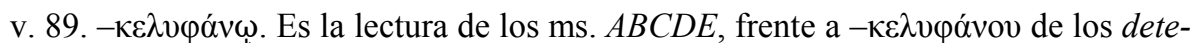
riores (véase nuestro comentario a la lectura adoptada en el verso 55). Además, opino, con A.Hurst, que es más esperado un instrumental en dativo, ya que - $\sigma \tau \rho o ́ \beta 1 \lambda o v$ es un acusativo de relación.

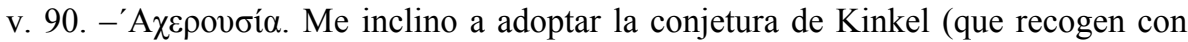
espíritu suave inicial los ms. $C D E$ ), antes que la corrección de Müller ( $\dot{\eta}$

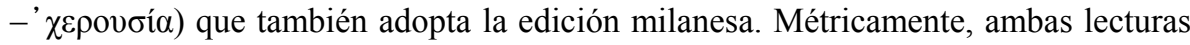
son correctas.

v. 100. $-\pi \lambda \eta \mu v p i ́ \delta o \varsigma$. Prefiero esta opción ortográfica que presentan los ms. AB antes que $-\pi \lambda \eta \mu \mu v p i ́ \delta o \varsigma$, opción propuesta por L.Mascialino.

v. 103. - yovaîv. Cf. el criterio de adopción dialectal a favor del jonio siempre que la tradición manuscrita presente variantes de otros dialectos, tal como queda explicado en el comentario al verso 2 .

v. 137. $-\theta \varepsilon ́ \mu t v$. Subscribo las palabras de A.Hurst al respecto (op.cit., p.55): «Chiamare - $\Theta \varepsilon ́ \mu ı \varsigma$ senza perifrasi è poco nello stile di Licofrone, cf. anche il verso 369)».

v. 139. $-\dot{\varepsilon} \varsigma$. Para la elección de esta lectura en vez de $-\varepsilon i \zeta$, véase mi comentario al verso 2.

v. 185. Post 185 no considero necesario suponer la existencia de una laguna para la mejor intelección del texto, ya que, entre otras cosas, el relativo femenino - १๊ v del v. 186 concuerda perfectamente con $-\pi$ ó $\rho$ vv, dos versos más arriba. Además, contamos con un buen parangón en Alex. 470.

v. 193. -кро́кๆıб.. Cf. el comentario al v.2. 
v. 210. $-\pi \tau o \lambda \iota \rho \rho \alpha i ́ \sigma \tau o v$. Para la elección de esta lectura en vez de $-\pi \circ \lambda \iota \rho \rho \alpha i ́ \sigma \tau o v$, véase mi comentario al verso 2 .

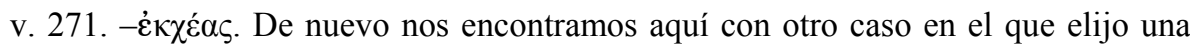
variante manuscrita considerada preferible. Si me decanto, como Hurst, por - $\dot{\varepsilon} \kappa \chi \dot{\varepsilon} \alpha \varsigma$ (ms. $A C D E$ ), en vez de $-\dot{\varepsilon} \gamma \chi \varepsilon \dot{\varepsilon} \alpha \varsigma$ (ms. $B$, lectura elegida por Mascialino), es porque el contexto hace pensar más en «extraer» que en «verter».

v. 271. - $\alpha \hat{v} \theta 1 \varsigma$. Para la elección de esta lectura en vez de - $\alpha \hat{\theta} \theta 1 \varsigma$, véase mi comentario al verso 2 .

v. $279 .-\dot{\varepsilon} \varsigma$. Cf. comentario al v.2 (para $-\dot{\varepsilon} \varsigma$ en vez de $-\varepsilon \dot{\varepsilon} \varsigma)$.

v. 346. $-\dot{\varepsilon} \kappa \pi \varepsilon \pi \lambda \varepsilon v \kappa o ́ \sigma$. Prefiero esta lectura (uulg.), como Hurst a la adoptada en el

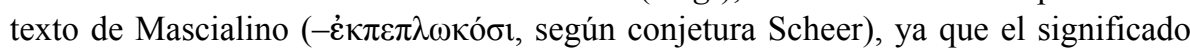
de la lectura adoptada, a saber, «para los que llegan por mar» (perf. - $\dot{\varepsilon} \kappa \pi \varepsilon ́ \pi \lambda \varepsilon v \kappa \alpha)$ hace innecesaria la conjetura.

v. 358. - $\ddot{\alpha} \rho \pi \alpha \varsigma$. Elijo una variante manuscrita que considero preferible. Si me decanto por $-\ddot{\alpha} \rho \pi \alpha 1 \varsigma$ (con Mascialino) en vez de $-\ddot{\alpha} \rho \pi \eta \varsigma$ (Hurst) es por la desinencia de la primera lectura, de acuerdo con el adjetivo con el que concuerda plausiblemente en dativo $(-\gamma \alpha \mu \psi \alpha \hat{\imath} \sigma \mathrm{v})$.

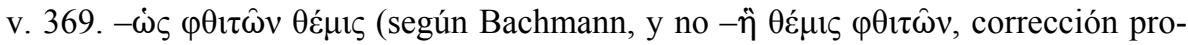
puesta por Kinkel y adoptada por Mascialino). Véanse las apreciaciones aducidas en el v.137 para entender la elección de esta conjetura.

v. 397. $-\sum \varepsilon$ cí́ov. Prefiero la lectura de los ms. $A D E T^{1}$, teniendo en cuenta, por lo demás, el parangón, destacado por A. Hurst, de genitivo con $-\dot{\alpha} \kappa \tau i ́ \varsigma$ en Píndaro, $-\Pi \alpha$

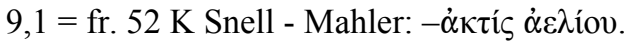

v. 398. - - $\alpha \pi \rho$ òv. Lección proporcionada por los deteriores codices, adoptada por Mascialino y no por Hurst, que preferiero a $-\sigma \alpha \theta \rho o ̀ v ~(-A B C D E)$ por cuanto el significado de la primera variante («pútrido en salazón») se adecúa perfectamente a

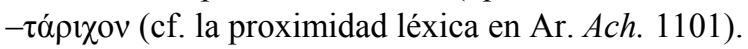

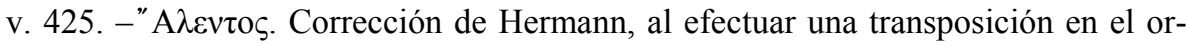




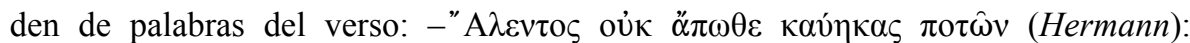

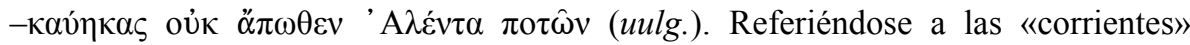
$(-\pi \mathrm{o} \tau \hat{\omega} v)$ parece lógico esperar en genitivo el nombre del río (en este caso el Ales). Cf. Estrabón, XIV 642; Pausanias V 28,3.

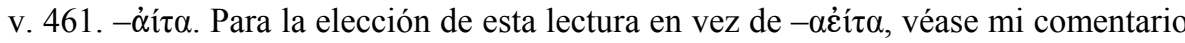

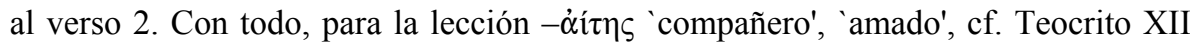
14,20 y $A P \mathrm{XV} 26$.

v. 480. - $\dot{\varepsilon} \gamma \gamma o ́ v \omega v$. Prefiero esta opción ortográfica que presenta el ms. B antes que

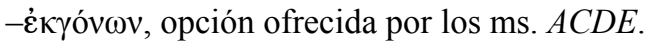

v. 520. - $\beta i ́ \alpha$. Aunque no hay variantes manuscritas, podemos interpretar que

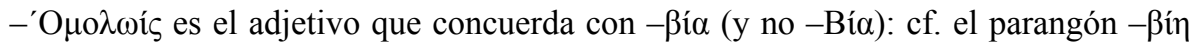

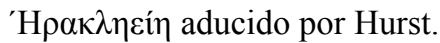

v. 533. $-\varepsilon \cup ̉ \pi \rho \varepsilon \pi \varepsilon i \varsigma$. Conjetura de Scheer (mientras que contamos como otras leccio-

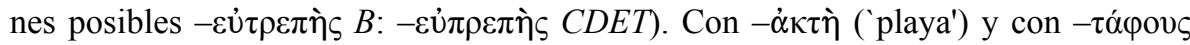

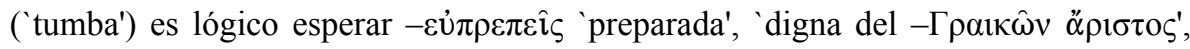

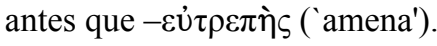

v. 542. $-\mu \varepsilon \imath \lambda i ́ \sigma \sigma \omega \sigma ı v$. Hurst, que prefiere $-\mu \varepsilon \imath \lambda \hat{\imath} \xi \omega \sigma \imath$, aduce la forma $-\delta \varepsilon \dot{\xi} \omega \nu \tau \alpha \iota$ del verso 539, es decir, tres versos más arriba. Con todo, prefiero, como Mascialino, la variante $-\mu \varepsilon \imath \lambda i ́ \sigma \sigma \omega \sigma \imath$, del verbo $-\mu \varepsilon \imath \lambda i ́ \sigma \sigma \omega$ ('calmar, apaciguar') por cuanto no creo necesario el tiempo futuro porque ello supone una regularidad estilística y lingüística que en todo momento Licofrón intentó descuidar ex profeso.

v. 546. $-\alpha \hat{\theta} \theta \mathrm{t} \varsigma$. Me veo inclinado a adoptar esta lectura tradicional de los ms. DET ${ }^{1}$ (en vez de - $-\alpha \hat{\tau} \tau ı \varsigma$ ), ya que contamos con un buen parangón en Sófocles, O.C. 364. De otro lado, su sentido ('otra vez', 'nuevamente', etc.) se corresponde al del verso licofroniano.

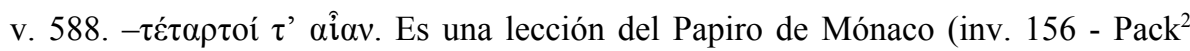
1286; ed. pr. Hartmann, Philologus, 76, 1920, pp. 228-233) que Mascialino no tuvo en cuenta y que sí recoge Hurst. Por lo demás, Licofrón seguro que debía preferir, como en tantos otros casos, el término - $\alpha \hat{i} \alpha v$, típicamente épico y trágico. 


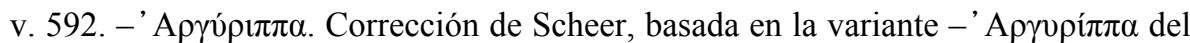
Cod. Marcianus 476, adoptada por Hurst y que yo mismo sigo en mi edición. Mas-

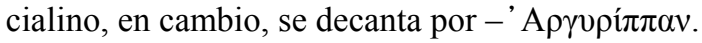

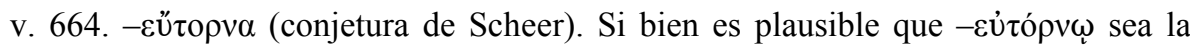
lección de $A C D E$ y que la interpretación de los escolios va en la misma línea, más

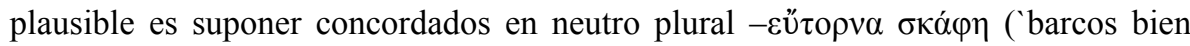
labrados').

v. 686. $-\Pi \varepsilon \mu \varphi$ í $\gamma \omega v$. Lectio difficilior (ms. $B$ ), en vez de $-\pi \varepsilon \mu \varphi$ í $\delta \omega v$, que adopto como genitivo complemento de -ö $\pi \alpha$.

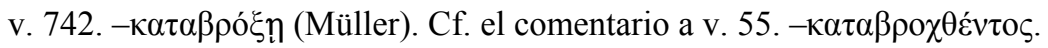

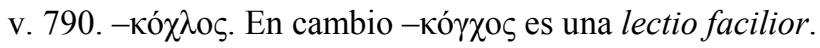

v. 816. $-\beta o \eta \lambda \alpha \tau o ̂ ิ v \tau$ (ms. $C D E$ ). La concordancia con el dativo $-\sigma o t$ del v. 815 me

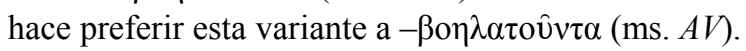

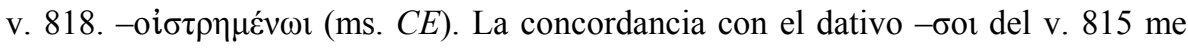
hace nuevamente preferir esta variante a la presentada por los ms. $A B D$, a saber,

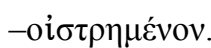

v. 841. - $\varphi \alpha ́ \lambda \lambda \alpha$ ava. Prefiero esta opción ortográfica que presentan los ms. $A C$, antes

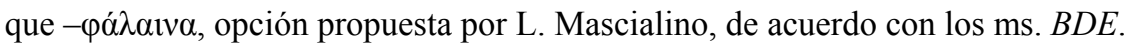

v. 843a. Post 843 no considero necesaria ni plausible la transposición (recogida en la edición de A. Hurst) después del v. 843, habida cuenta la coherencia interna del hilo narrativo y el principio o el final de los versos anteriores y posteriores.

v. 844. - $\dot{\text { o. }}$ Cf. el comentario ad 845.

v. 845. $-\pi \dot{\varepsilon} \tau \rho \omega v$. Adopto esta variante (ms. CDET) que ya escogió Mascialino en su edición teubneriana de 1964 (si bien en la primera edición publicada en Alma Mater, Madrid, 1956, leemos $-\pi \varepsilon ́ \tau \rho \omega$, conjetura de Scheer). El motivo de la elección es su dependencia con el dativo $-\dot{\alpha} \mu \varphi \varepsilon \lambda v \tau \rho \omega ́ \sigma \varepsilon 1$. 
v. 848. $-\chi \alpha \mu \varepsilon v v \alpha ́ \delta \omega v$. Además de ser lectio difficilior, esta variante la vemos más plausible por su dependencia como genitivo con-Eủvás del siguiente verso.

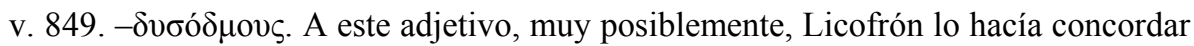
(hipálage) con un sustantivo que no le correspondía.

v. 853. $-\Sigma \kappa v \lambda \eta \tau \rho i ́ \alpha$ ( $u$ ulg. y atestiguado por Eustacio). Por su parte, Mascialino prefiere la conjetura de Scheer, a saber, $-\Sigma \kappa v \lambda \lambda \eta \tau \imath \alpha$.

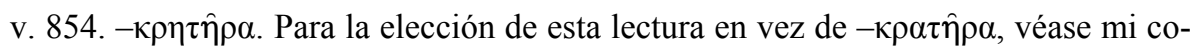
mentario al verso 2 .

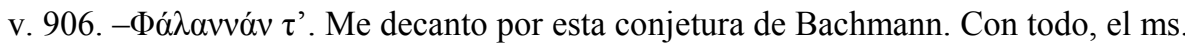
$A$ ofrece $-\Phi \alpha ́ \lambda \alpha v v o ́ v ~ \tau$ ', del que afirma Hurst, posiblemente con razón: «un toponimo su cui esiste, evidentemente, un'esitazione sul genere; non si perde niente dunque a conservare il testo dei manoscritti di Licofrone».

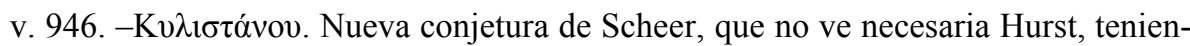

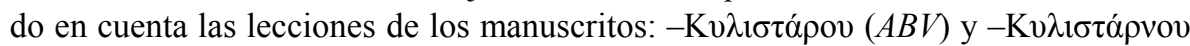
$(C D)$.

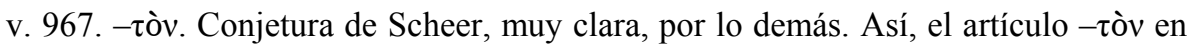

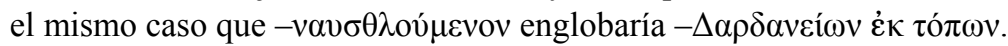

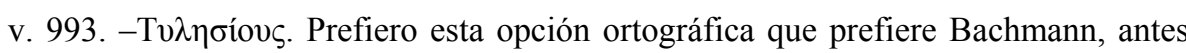

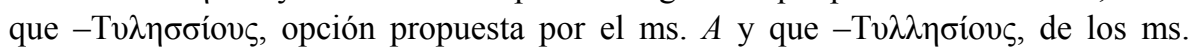
$B C D E$.

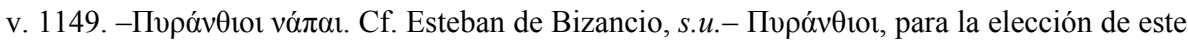
topónimo derivado.

v. 1157. -ǒ $\tau \alpha v$. Me decanto por la conjetura tradicional de Hermann (que presentan

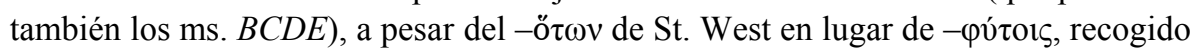
por Hurst en su edición milanesa.

v. 1159. No considero necesaria ni plausible (aunque no imposible) la transposición (a cargo de St. West) después del v. 1173, habida cuenta la coherencia interna del 
hilo narrativo de los versos anteriores y posteriores, así como el principio y el final de los mismos.

v. 1162. $-\kappa \dot{\alpha} \kappa \kappa \varepsilon ́ \lambda \varepsilon v \theta \alpha$. Variante presentada por los ms. $C D$. Con todo, $-\kappa \dot{\alpha} \kappa \varepsilon \dot{\lambda} \varepsilon \varepsilon v \theta \alpha$ es una lección del Papiro de Mónaco (inv. $156-$ Pack $^{2}$ 1286; ed. pr. Hartmann, Philologus, 76, 1920, pp. 228-233) que Mascialino no tuvo en cuenta y que sí recoge Hurst. Mi elección se basa en la lección que nos ofrece otro manuscrito, el $E$, muy semejante: $-\kappa \alpha \kappa \kappa \varepsilon ́ \lambda \varepsilon v \theta \alpha$.

v. 1179. $-\sigma \varepsilon \dot{\beta} \omega \sigma \mathrm{l}$. Variante recogida por los ms. $B E$ que prefiero, como Hurst, aduciendo su utilización en los trágicos sin la partícula - đ̌v.

v. 1187. $-\pi \rho \hat{\omega} \tau o v$. Prefiero relacionar el numeral cardinal con $-\pi \dot{\varepsilon} \tau \rho o v$, tal y como lo presentan los ms. $C D E$, a entender que va concordado con Ulises, como apunta Hurst, basándose en los ms. $A B$.

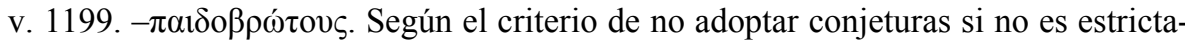
mente plausible y necesario para la intelección del texto, prefiero conservar esta lec-

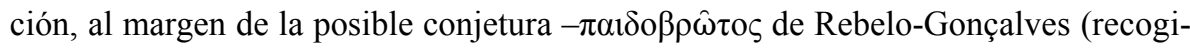
da también por Hurst). Cf. el artículo - $\tau \dot{\alpha} \varsigma$.

v. 1219. $-\psi v \delta \rho \alpha i ̂ \iota v$. Hurst afirma: $-\psi v \delta \rho \alpha i ̂ \sigma ı ~ \tau$ ' («la particella connette i due participi»), y lo corroboran los ms. $A B D E$. Con todo, Scheer conjetura - $\psi v \delta \rho \alpha \hat{\sigma} \sigma v \sin$ partícula alguna, concordado con $-\mu \eta \chi \alpha v \alpha i ̂ s$.

v. 1219. $-\dot{\alpha} v \alpha \varphi \lambda \dot{z} \gamma \omega v$. Sigo la conjetura de Scheer, a pesar de las indicaciones de Holzinger postulando la forma $-\dot{\alpha} v \alpha \pi \lambda \dot{\varepsilon} \kappa \omega v$ (uulg.).

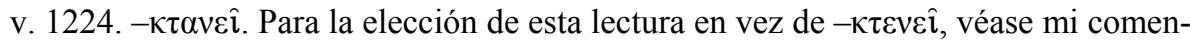
tario al verso 2 .

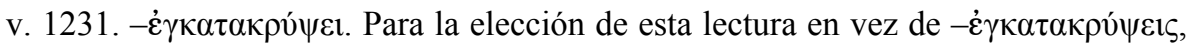
véase mi comentario al verso 2 .

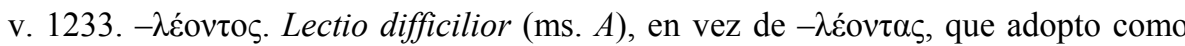
genitivo complemento de $-\sigma \kappa u ́ \mu v o u \varsigma$. 
v. 1234. $-\tau \hat{\eta} \varsigma \tau \varepsilon$ X $\varepsilon 1 \rho \alpha ́ \delta$ os. Adopto esta variante toponímica (ms. $A D E$ y sin el $-\tau \varepsilon$, también $C$ ) que ya escogió Mascialino en su edición teubneriana de 1964 (si bien en la primera, Alma Mater, Madrid, 1956 leemos - $\tau \varepsilon$ Xoıpódos, conjetura de Canterus).

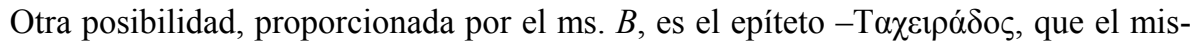
mo Hurst trata de explicar etimológicamente.

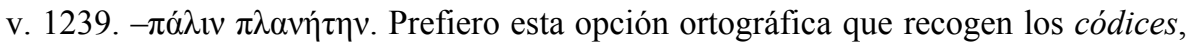
antes que $-\pi \alpha \lambda \uparrow \mu \pi \lambda \alpha v \eta ́ \tau \eta \nu$, opción que prefieren algunos editores de acuerdo con Steph. Byz., s.u. -’A $\lambda \mu \omega \pi i ́ \alpha:-\pi \alpha ́ \lambda$ เv $\pi \lambda \alpha v \eta \dot{\tau} \eta v$.

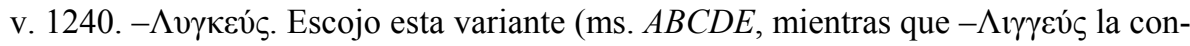
templan los deteriores), consciente de la acostumbrada capacidad de Licofrón para escoger la versión mítica y onomástica menos frecuente.

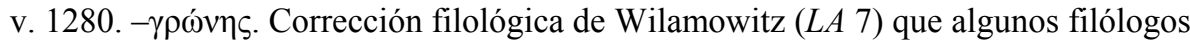
posteriores (como el mismo Mascialino) no adoptaron. Con todo, mi elección la veo corroborada al leer Alex. v. 631, en el que aparece $-\gamma \rho \hat{\omega} v o v$.

v. 1280. Post 1280 no considero necesaria la posibilidad de la existencia de una laguna (como sí, en cambio, propuso Scheer) para la mejor intelección o coherencia interna del texto, teniendo en cuenta el fin del parágrafo y el principio (plausible a

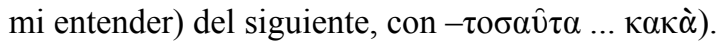

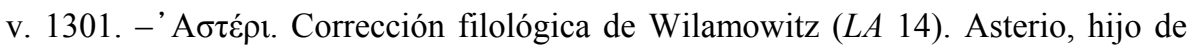
Téctamo, era rey de la isla de Creta.

v. 1366. $-\alpha \hat{\theta} \theta 1 \varsigma$. Me inclino a adoptar esta lectura tradicional de los deteriores (en vez de - $\alpha$ ¿ıৎ), según lo expresado en los vv. 546 y 1431.

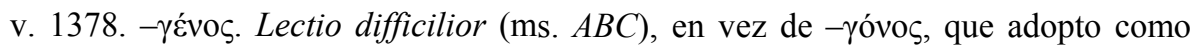
«acusativo de relación» (cf. Od. XV 257).

v. 1391. $-\Lambda \eta \kappa \eta \tau \rho i ́ \alpha$. Me decanto por la corrección filológica de Schwyzer (RM 75, p.448) en vez de $-\lambda \eta \kappa \tau \eta \rho i ́ \alpha v$ (de los ms., preferida por el editor Mascialino) por

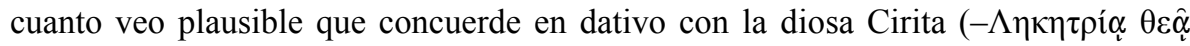

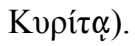


v. 1403. $-\alpha i \mu$ o $\omega ́ \tau \alpha \iota \sigma v$. Escojo esta lectura en vez de la propuesta de Pfeiffer (y de

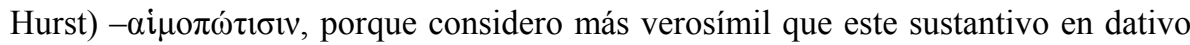
plural tenga su nominativo singural en - $\alpha i \mu$ o $\omega \dot{\tau} \eta \varsigma$ ('chupadora de sangre'), en re-

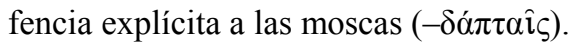

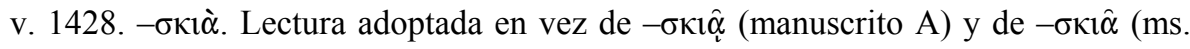

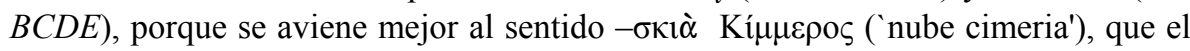
mismo G. Paduano traduce por «nebbia cimmeria», aunque A. Hurst, coeditor de la

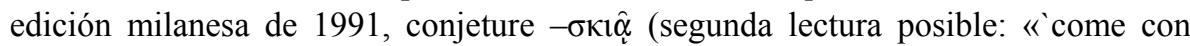
dell'ombra', perché sono le frecce che nasconderebbero il sole e mimerebbero l'ombra», p. 56).

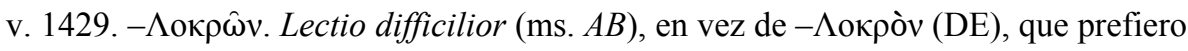
de acuerdo con el parangón de los Paroemiographi Graeci, t. 1, p. 116 y t. 2, p. 186, según indica sagazmente Hurst en su edición (p. 56). Por lo demás, esta lectura no altera métricamente el verso.

v. 1431. $-\alpha \hat{\theta} \theta$ is. Me veo inclinado a adoptar esta lectura tradicional de los ms. DE (en vez de - $-\hat{v} \tau \imath \varsigma$ ), ya que contamos con un buen parangón en Sófocles, O.C. 364. De otro lado, su sentido ('otra vez', 'nuevamente', etc.) se corresponde al del verso licofroniano que comienza por $-\alpha \hat{\theta} \theta \iota \varsigma \alpha \lambda \lambda \mu \pi \lambda \omega ́ \tau o เ$...

v. 1436. -Airaíaıs. Lectura preferida de acuerdo con U. v. Wilamowitz (De Lycophronis Alexandra commentatiuncula, Greifswald, 1883, p. 7), en vez de otras posi-

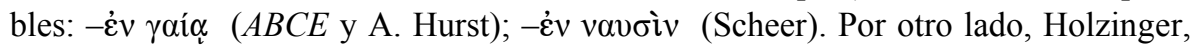
en su edición de 1895 , se decantó por la primera posibilidad, aunque con una ligera modificación, proponiendo-Airaías.

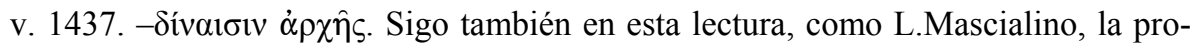
puesta de U. v. Wilamowitz (De Lycophronis Alexandra ..., p. 7), en vez de la que aparece en los manuscritos, a saber, $-\delta \varepsilon ı \alpha \hat{\imath} \sigma ı v \dot{\alpha} \rho \chi \alpha \hat{\imath} \varsigma$, lectura adoptada también por Hurst. Con todo, el mismo Holzinger se inclina de nuevo por la primera posibilidad ya conjeturada, si bien con la variante $-\dot{\alpha} \rho \chi \grave{\alpha} \varsigma$ en vez de $-\dot{\alpha} \rho \chi \hat{\eta} \varsigma$.

v. 1438. $-\dot{\alpha} \lambda$ ó . Me he decidido por esta lectura en vez de $-\chi \theta$ ovó $\varsigma$ (cf. Euphrosyne, N.S. XVI, 1988, pp. 247-255), aunque podría argumentarse en favor de la segunda 


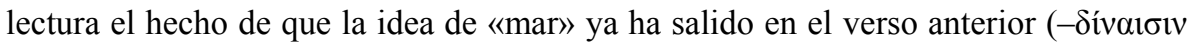
...) y que, por tanto, puede haber un cierto pleonasmo.

v. 1444. $-\Gamma \alpha \lambda \alpha ́ \delta \rho \eta \zeta$. Jonismo en vez de $-\Gamma \alpha \lambda \alpha ́ \delta \rho \alpha \varsigma$ (cf. nuestro comentario a la lectura adoptada en el v. 2).

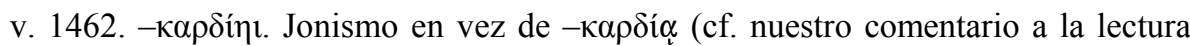
adoptada en el v. 2).

Josep ANTONI Clua SERENA 